DHPARTAMENTO DF: QUMMCA ORGANICA F MIOIGGICA

Diretor: Prof. Dr. Fansecer Kibeiro

\title{
SÓBRE O TEÔR DE ẢCIDO ASCôRBICO NO SANGUE TOTAL DE EQÜINOS (P.S.) DA VILA HÍPICA DO JOCKEY CLUB DE SÃO PAULO
}

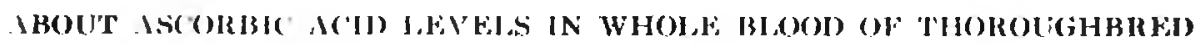 \\ RACW HORSIES
}

ARNaldo Costa

Assistente
0. F. Ribeino

Tecnologista

()correu-nos determinar estatisticamente a taxa de ácido ascórbico no sanỹue de eqüinos, por não haver até o presente momento dados a respeito que possam ser utilizados como valôres médios em animais clìnicamente sãos.

Para lal realizar escolhemos os animais alojados na Vila Hípica do Jotkey Club de São Paulo. constituindo um conjunto homogêneo no que diz res. peito à raça - puro sangue de corrida - alimentação e sistema de trabalho, além de estarem sob contrôle clinico permanente.

Sendo o regimern alimentar aproximadamente idêntico, de ordem quantitativa. pode-se considerá-lo como padronizado.

Quanto ao trabalho. majs on menos sistemático. consta de exercícios preparatórios de pista, em geral pelo jeriodo da manhā e de exercícios leves. como passeios durante alguns minutos no pátio onde se acham localizadas as cochei ras, pelo período da tarde.

Compöe-se a alimentação de rações constituidas de milho, aveia. cenoura e elententos forrageiros verdes. como grama e kikuio, além de alfafa. esta en prequena proporçăo.

I quantidade media diária administrada a cada animal era a seguinte:

\begin{tabular}{|c|c|c|}
\hline Ivei: & & 6 quiles \\
\hline Milho & & fi quilos \\
\hline Cenoura & & 0,5 a 0,8 quilu \\
\hline Verdes & & 0,5 it \\
\hline
\end{tabular}


Das análises procedidas nos elementos componentes da ração, verificamos os seguintes índices médios: (")

\begin{tabular}{|c|c|c|c|c|}
\hline & & Scido & $\begin{array}{c}\text { inscúrbico } \\
\text { M }\end{array}$ & $(m g e)$ \\
\hline A veia & ( 5 amostris) & 0,118 & $(0,08$ & $-\quad 0,26)$ \\
\hline Milho & ( 5 anrostras) & 0,000 & $-(-$ & ) \\
\hline Cenours & (10 amostras) & 5,104 & $-(4,40$ & - $\quad 5,6 t)$ \\
\hline Kikuio & (20 amostras) & 51,970 & $-(27,10)$ & ․ 79,06) \\
\hline Grâma & (20 amostras) & 46,056 & $-(26,6.3$ & $-73,42)$ \\
\hline Alfafi & $(5$ anostras $) \ldots \ldots$ & 6,200 & $-(5,50$ & $6,80)$ \\
\hline
\end{tabular}

Dos resultados obtidos pelas análises, destacam-se os elementos que encer. ram maior teôr em ácido ascórbico, que são justamente aqueles que entram em menor proporção na composição da ração diária.

\section{MATERIAS, F, METOIOO}

Os animais utilizados para as análises constituiam lote hastante homogêneo quanto ao pêso, que variava de 400 a 420 quilos, sendo os limites de idade correspondentes de 2 a 8 anos. com predominância de exemplares entre 2 e 5 anos.

0 sangue retirado mediante punçĩo da veia jugular. con agulta apropria. da. era colocado em tubo contendo anti-coagulante sêco loxalato de sídio a $3 \%)$ em quantidade suficiente para o volume desejado $(10 \mathrm{ml})$. sendo a seguir agitado e levado ao laboratónio para a respectiva análise.

O método utilizado o de Roe e Kuether, método colorimétrico esquenratizado adiante - -., foi o escolhido para as determinaçōes em sangue total.

a) - Desproteinizą̧ăo

1) - Num tubo de centrifuga de $50 \mathrm{ml}$ colocar:

$15 \mathrm{ml}$ de ácido tricloroacético a $6 \%, 5 \mathrm{ml}$ de sangue total. gôta a gôta, agitando com um bastāo de vidro com a finalidadr de se obter uma mistura bem fina.

(*) () método empregado parit esta dosagem foi o publicado na Revista da Faculdade de Medicina Veterinária, vol. $\mathrm{n}^{\star 2}$, fasc. 2, setembro 1942, págs. 29/39. 
2) - Deixar pelo menos 5 minutos em repouso e centrifugar.

3) - O liquido sobrenadante é recolhido a um frasco, contendo carvāo ativado $(0,75 \mathrm{~g}$ aproximadamente); agitar vigorosamente.

4) Filtrar em papel de filtro de $9 \mathrm{~cm}$ de diâmetro.

b) - Desenvolvimento de côr a fotometria

5) - Tomar dois tubos de 25x105, A e B. Acrescentar a ambos $4 \mathrm{ml}$ do filtrado e uma gôta de uma solução alcoólica de tiouréa a $10 \%$.

6) - Acrescentar a A, $1 \mathrm{ml}$ de 2,4-dinitrofenilhidrazina em soluçäo a $2 \% \mathrm{~cm}$ ácido sulfúrico a $85 \%$. colocar em banho-maria a $37^{\circ} \mathrm{C}$, durante 3 horas exatamente; colocar igualmente em banho-maria o tukio B.

7) - Retirar $A$ e $B$ do banbo-maria f colocá-los em água gelada contendo gệlo em abundância.

81 - Acrescentar a A c a $B .5 \mathrm{ml}$ de ácido sulfúrico a $85 \%$. A adição deve ser lentu por meio de uma bureta graduada, uma gôta de cada vez, com agitação dentro do banho gelado; levar aproximadamente 1 minuto, para que seja adicionado todo o reagente; não lubrificar a torneira da burcta com vaselina.

9) - Acrescentar a B $3 \mathrm{ml}$ de 2,4-dinitrofenilhidrazina. Agitar ambos os tubos no banho de água gelada. Passar para uma estante para lubo de ensaio.

(1i) Depuis de 30 minutos em repouso. ler no fotometro, utilizando o filtro verde ou 5.10 .

\section{R MSET TAUUS}

O: valores the icido ascórhico encontrados em cavalos puro sangue de corrida, computados para ambos os sexos, sĩo apresentados na tabela I. 
TABELA I - TEORES DE NCIDO ASCORBICO FM SANGUE 'TO'AAT I)E EQt'INOS

\begin{tabular}{|c|c|c|c|c|c|}
\hline $\mathbf{N}^{0}$ & $\begin{array}{c}\text { Acido } \\
\text { ¿scórbicu } \\
\text { mg\% }\end{array}$ & No & $\begin{array}{c}\text { Ácido } \\
\text { ascórbicos } \\
\text { mgrer }\end{array}$ & Nv & $\begin{array}{l}\text { icido } \\
\text { ascórbicı) } \\
\text { inger }\end{array}$ \\
\hline 1 & 0,680 & 40 & $0,4: 20$ & 79 & 0,925 \\
\hline 2 & 0,580 & $H 1$ & 0,500 & 80 & 0,840 \\
\hline 3 & 0,680 & 12 & 0,525 & 81 & 13,620 \\
\hline 4 & 0,510 & 43 & 0,435 & 83 & $(1,600$ \\
\hline 5 & 0,560 & 44 & 0,170 & 83 & 0,565 \\
\hline ii & 0,420 & 45 & 0,740 & 84 & $(0,810$ \\
\hline 7 & 0,690 & 16 & $0,7 \% 0$ & 85 & 10,790 \\
\hline 8 & 0,635 & 47 & 0.7601 & 86 & 0,860 \\
\hline 9 & 0,435 & 48 & 0,6211 & ST & 0,785 \\
\hline 10 & 0,575 & f!! & 0,050 & 88 & 0,530 \\
\hline 11 & 0,530 & 50 & 0,620 & $8 !$ & 0,730 \\
\hline 12 & (0,575) & 51 & $0,7 \geq 01$ & 90 & $(1,760$ \\
\hline 13 & 0,700 & $5: 2$ & $1), 8,311$ & 91 & 0,780 \\
\hline 14 & $0, f ; 40)$ & 5is & $(1,650)$ & 92 & 0,680 \\
\hline 15 & 0,565 & 51. & $0,4,00$ & 933 & 0,660 \\
\hline 16 & 0,610 & 55 & $01,3: 30$ & 94 & $0,7 \pi 0$ \\
\hline 17 & 0,720 & 56 & 0,510 & $9: 5$ & 0,880 \\
\hline 18 & 0,725 & 57 & 0,780 & $9 t i$ & 0,690 \\
\hline 19 & 0,610 & is & 0,680 & $9 \tau$ & 0,710 \\
\hline 20 & $0,63.5$ & 59 & $10,6 \%()$ & $9 \pi$ & 0,570 \\
\hline 21 & $0,685)$ & (i) & $(0, \bar{s} !)$ & $9 !$ & 0,5330 \\
\hline 22 & $0,8 \div 0$ & (i) & 0,380 & 100 & $(1,6 \div 0$ \\
\hline 23 & 0,6230 & 62 & $0,10()$ & 101 & 0,770 \\
\hline 21 & 0,505 & ti:3 & $0,1.10$ & 102 & 0,710 \\
\hline 25 & 0,600 & 64 & $(0,33)$ & 103 & 0,420 \\
\hline 26 & 0,795 & (i.) & 0,550 & 10.4 & $0,(640)$ \\
\hline 27 & 0,650 & $6 t i$ & $(1,140)$ & 105 & 0,615 \\
\hline 28 & 0,610 & 6i & $(0,4,20$ & $10 f i$ & 0,600 \\
\hline 29 & 0,550 & 68 & $(1,5: 311$ & .107 & 0,680 \\
\hline$: 30$ & 0,390 & (i) & 0,770 & 108 & 11,605 \\
\hline 31 & 0,5440 & 70 & 0,710 & $10 ! 1$ & $0, f f(1)$ \\
\hline 32 & 0,4811 & $\gamma_{1}$ & 0,970 & 111 & 0,510 \\
\hline 33 & 0,510 & 72 & $(1,920)$ & 111 & $0,6: 20$ \\
\hline 31.1 & 0,410 & $7: 3$ & 0,860 & 112 & 0,445 \\
\hline 35 & 0,7810 & 71 & 0,795 & 113 & $0,6: 10$ \\
\hline $36 i$ & $(0,610)$ & $7: 5$ & 0,795 & 114 & $(1,5440$ \\
\hline $3 \pi$ & 0,790 & 76 & $(0,705)$ & 115 & $(0,590$ \\
\hline 38 & 0,760 & $7 \pi$ & 0,920 & 116 & $(1,560$ \\
\hline 39 & 0,675 & 78 & 0,770 & 117 & 0,500 \\
\hline
\end{tabular}

Como serifica. a mielia glohal em 117 animais 169 machos of 18 fèmeas) cquivale a $0,6 \% 3 \pm 0,013 \mathrm{mg}$ per $100 \mathrm{ml}$ de sangue total. Os limites da dis. tribuiçāo das frequências correspondem respectivamente às observações de núme. ros 64 e $5.510,3.30 \mathrm{mg}$ por $1010 \mathrm{ml}$ de sanguel e $71.0 .970 \mathrm{mg}$ por $100 \mathrm{ml})$. 
() dois primeiros valôres estabelecidos em um macho e uma fêmea, o último teterminado em um macho.

A amplitude de variação atinge pois a $0,640 \mathrm{mg}$ por $100 \mathrm{ml}$ de sangue lontal.

Sa tahela Il achan-se reunidos os indices calculados para os machos. isoladamente.

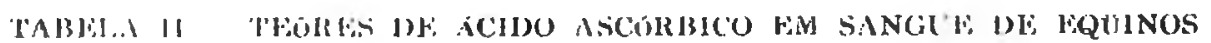

$$
M \text { a c ll os }
$$

\begin{tabular}{|c|c|c|c|c|c|}
\hline N" & $\begin{array}{l}\text { Acido } \\
\text { ascórbico } \\
\text { mg\%. }\end{array}$ & $N^{*}$ & $\begin{array}{l}\text { Acido } \\
\text { iscórbico } \\
\text { mg\% }\end{array}$ & No & $\begin{array}{l}\text { Acido } \\
\text { ascúrbico } \\
\text { mg\% }\end{array}$ \\
\hline 4 & & 19 & 0595 & & \\
\hline 5 & 0,560 & $\begin{array}{l}+2 \\
43\end{array}$ & $\begin{array}{l}1,520 \\
0,435\end{array}$ & 75 & $\begin{array}{l}0,795 \\
0,795\end{array}$ \\
\hline 7 & 0,690 & 44 & 0,470 & 76 & 0,795 \\
\hline $\mathbf{s}$ & $0,6 j 35$ & 45 & 0,710 & 78 & 0,770 \\
\hline 111 & 0,575 & .46 & 0,770 & 79 & 0,925 \\
\hline 12 & 0,575 & 47 & 0,760 & 82 & 0,600 \\
\hline 14 & $0,6+40$ & 49 & $0,6.111$ & 83 & 0,565 \\
\hline 15 & $0,5(2)$ & .50 & 0,620 & 85 & 0,790 \\
\hline 17 & $0, \pi \geqslant 0$ & 51 & 0,72010 & 86 & 0,860 \\
\hline 18 & 0,5025 & 52 & $(1,830$ & $s s$ & 0,530 \\
\hline 19 & 0,610 & it & 0,170 & 9.1 & 0,770 \\
\hline$\because 1$ & 0,685 & 55 & 0,3330 & 96 & 0,600 \\
\hline 22 & 0,810 & 56 & 0,510 & 96 & 0,570 \\
\hline 27 & 0,650 & xi & $0,780 !$ & 10.4 & $0,6 \cdot 10$ \\
\hline 30 & 0,390 & 60 & 0,560 & 105 & 0,615 \\
\hline 32 & 0,180 & 65 & $0,5.50$ & 1013 & 0,690 \\
\hline 34 & 0,410 & $b l i$ & $0,4 \cdot 10$ & $16 !$ & 0,440 \\
\hline 35 & 0,780 & 67 & 0,420 & 110 & 0,510 \\
\hline 37 & 0,790 & is & 0,830 & 112 & $0,+115$ \\
\hline 38 & 0,760 & 69 & 0,770 & 113 & 0,640 \\
\hline 39 & 0,675 & $\pi 1$ & 0,970 & 114 & $0,51.10$ \\
\hline 40 & $0,4 \geq 0$ & 72 & 0,920 & 115 & 0,590 \\
\hline 41 & 0,500 & 73 & 0,860 & 117 & 0,500 \\
\hline \multicolumn{6}{|c|}{$\begin{array}{r}\mathrm{M}=0,638 \mathrm{mg} \\
\sigma=0,146 \mathrm{mg} \\
\sigma v-0,(1) 7 \mathrm{mg}\end{array}$} \\
\hline
\end{tabular}

1 midia ralculada separadamente en 69 machos. numa distribuiçāo que lun for valôre: míximox e mínimos. respectivamente, $0.970 \mathrm{mp}$ por $100 \mathrm{ml} \mathrm{de}$ sangul colal lobs. n* 7l) e 0,330 mg por $100 \mathrm{ml}$ de sangue cotal (obs. n 55). foi cocontradar a $0,638 \pm 0,017 \mathrm{mg}$ por $100 \mathrm{ml}$ de sangue cotal.

Vota-se prois que a amplitude de variação cifra-se en $(0,640 \mathrm{mg}$ por $100 \mathrm{ml}$

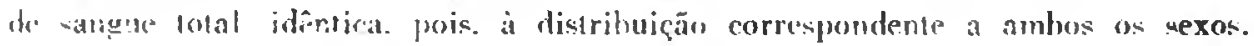


TABLSA III - TEORES DE ACIDO ASCORBICO FM SANGUE TUTAI.

DE EQUINOS - rêtme as

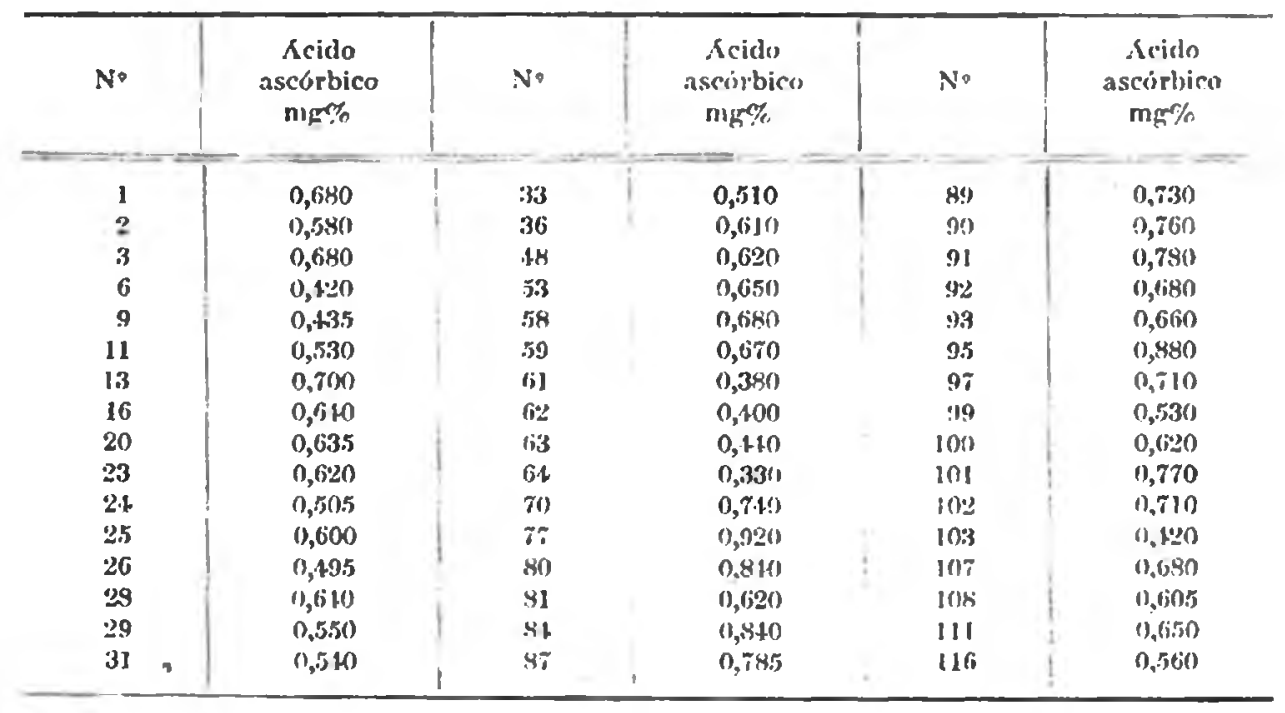

$$
\begin{aligned}
\mathbf{M} & =0,625 \mathrm{mg} \\
\sigma & =0,131 \mathrm{mg} \\
\sigma \nu & =0,019 \mathrm{mg}
\end{aligned}
$$

Na tabela III encuadramos os niveis relativos unicamente às fêmeas, em número de 48. A anplitude de variação está compreendida entre $0,330 \mathrm{mg}$ por $100 \mathrm{ml}$ de sangue total (obs. $\mathrm{n}^{\circ}$ 64) e $0.590 \mathrm{mg}$ por $100 \mathrm{ml}$ de sangue total (obs. $\mathrm{n}^{\circ} 77$ ), atingindo, pois, a $0,590 \mathrm{mg}$ por $100 \mathrm{ml}$ de sangue total. $A$ mé. dia desta distribuiçĩo é de $0,625 \pm 0,019 \mathrm{mg}$ por $100 \mathrm{ml}$ de sangue total.

Os niveis de $0,638 \pm 0,017$ e $0,625 \pm 0,019 \mathrm{mg}$ de atcido atscórhico por 100 $\mathrm{ml}$ de sangue intal, estabeleridos $\mathrm{cm}$ machos e fêmeas. afastados apenas 0.013 $\mathrm{mg}$ por $100 \mathrm{ml}$ de sangue, revclaram-se, quando sulmetidos ao test $t=0,19$, destituidos de significação estatística. Por essa razĩo, a média e reśpectivo êrro indicados na tabela I expressam-se perfeitamente na dosagem de ácido ascir. bico por $l(0) \mathrm{ml}$ de: sangue total em animais puro sangue de corrida, em regimem hípico.

\section{Msetsisto}

De acôrdo com os valôres detcrminados para os componentes da raçāo em teior de ácido ascórbico, o animal recehe diüriamente uma média de $560 \mathrm{mg}$ dessa vitamina. 
Considerando, ademais, que os eqüinos fazem parte do grupo de animais capacitados a sintetizar a vitamina $C$, pode-se admitir que a quantidade ingerida com os alimentos é suficiente para garantir-se um teôr que permita equilíbrio entre a ingestão e consumo de ácido ascrirbico em condiçōes normais de irabalho.

Ainda que não esteja bem definido o papel da vitamina $C$ : na alimentação dos eqüinos, certo número de pesquisadores supõe que o cavalo possa, como se disse, sintetizar vitamina $C$ para as suas próprias necessidades orgânicaş e, se. gundo Cassius WAY, pesquisas mais completas estão ainda por elucidar esta questão.

E" fóra de dúvida, entretanto, que em condições especiais, como periode de monta (Davis e CoLE), em condições de trabalho muscular intenso (Thomson) - certamente, no decurso de moléstias febris, o consumo de vitamina (: possa provocar grandes quedlas do teôr dessa substância no sangue.

Com efeito, Way pôde verificar a necessidade de se administrar vitamina (; como suplementação, em período de monta. ligando a ação dessa vitamina a motilidade dos espermalozóides. A administração diária de 1 grama da vitamina C durante êssc período, era suficiente: para equilibrar suas necessidades e manter o teôr normal no sangue.

Em periodo de trabalho muscular intenso, Tiromsos constatou, em equiinos, que apresentavam sintomas de azotúria, quedas tão grandes no têor de vitamina C no sangue, a ponto de encontrar animais com taxas iguais a $0,000 \mathrm{mg} \%$.

Reconhecida, pois, a importância da vitamina $C$ na alimentação dos equíinos e estabelecida a inexistência de dados sôhre a taxa normal dessa vitamina em sangue de eqüinos, mediante o exame da literatura ao nosso alcance, julgamos oportuno fixar os valôres normais de ácido ascórbico em apreciável número de espécimens, trabalhando com sangue total.

A propósito, cumpre referir que optamos pela determinação em sangue global, utilizando o método de Koe es Kuether, ao invés de trabalharmos ùnicamente com sôro ou plasma, pois, segundo os autores do método, o desaparecimento da vitamina $C$ em caso de subvitaminose, dá-se mais ràpidamente no plasmi do que nas células vermelhas e brancas; por outro lado, Cirinion verificou o mesmo fenômeno em relação is plaquetas. Conclui-se, assim, que a determinação será mais representaliva quando operada em sangue total.

Resulta da comparação dos valôres médios por nós estafielecidos, em san gue total, com os indicados por THOMson, trabalhando com sôro e plasma, e Davis e Cole, utilizando plasma, que os valôres médios determinados por êsses autores são menores em relação aos por nós encuntrados. 


\begin{tabular}{|c|c|c|c|c|c|}
\hline \multirow[b]{3}{*}{ 'I'Hомвок' } & \multicolumn{3}{|c|}{ Acido ascórbico (nıp\%) } & \multirow[b]{2}{*}{ Sanzue } & \multirow[b]{2}{*}{ total } \\
\hline & & & P'lasma & & \\
\hline & 0,8011 & 0,3430 & $0,720-0,170$ & + & $\cdots$ \\
\hline Inavis e Cold: .. & $-\infty$ & $\cdots$ & $0.700 \ldots 0,150$ & - & - \\
\hline A. $\quad$. $\ldots \ldots \ldots$ & - & $\cdots$ & .. & 0,970 & 0,33531 \\
\hline
\end{tabular}

Os valôres médios encontrados por 'THomson foram $0,533 \mathrm{mg} \%$ em sôro, utilizando 10 exemplares, enquanto, no plasma. trabalhando com 8 . a média foi de $0,588 \mathrm{mg}$ \%

Davis e Col.t, diante dos nossos resultados apresentam médias bem mais baixas e mesmo diante dos resultados apresentados por THOmson.

Pur outro lado, o confronto dos valôres médios por nós conseguidos em equiinos, com os indicados para o homem e para algumas espécies animais, nos proporciona interessantes ensinamentos.

Hawk e Bergeim reuniram valôres obtidos por Taylor, Chase e Fallknit en sangue humano que, confrontados com os por nós obtidos, são superioress, com média $1,610 \mathrm{mg}$ por $100 \mathrm{ml}$ de sangue total com variação de $0.8-2.4 \mathrm{mg}$ de ácido ascórbico.

Best e TAYlok conferem ao sangue humano o teôr médio de 0,5 ing de icido ascórbico por $100 \mathrm{ml}$ de sangue total. Este teôr em confronto com os por nós obtidos são menores.

Para algumas espécies animais podernos verificar pelo quadro adiante que. òmente as galinhas (Gallus gallus) apresentam valôres superiores aos conseguidos por nós, para os equiinos.

\section{ACIDO ASCORBICO NAS VARIAS ISPPECIES ANIMALS VAIOHLS

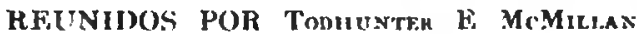

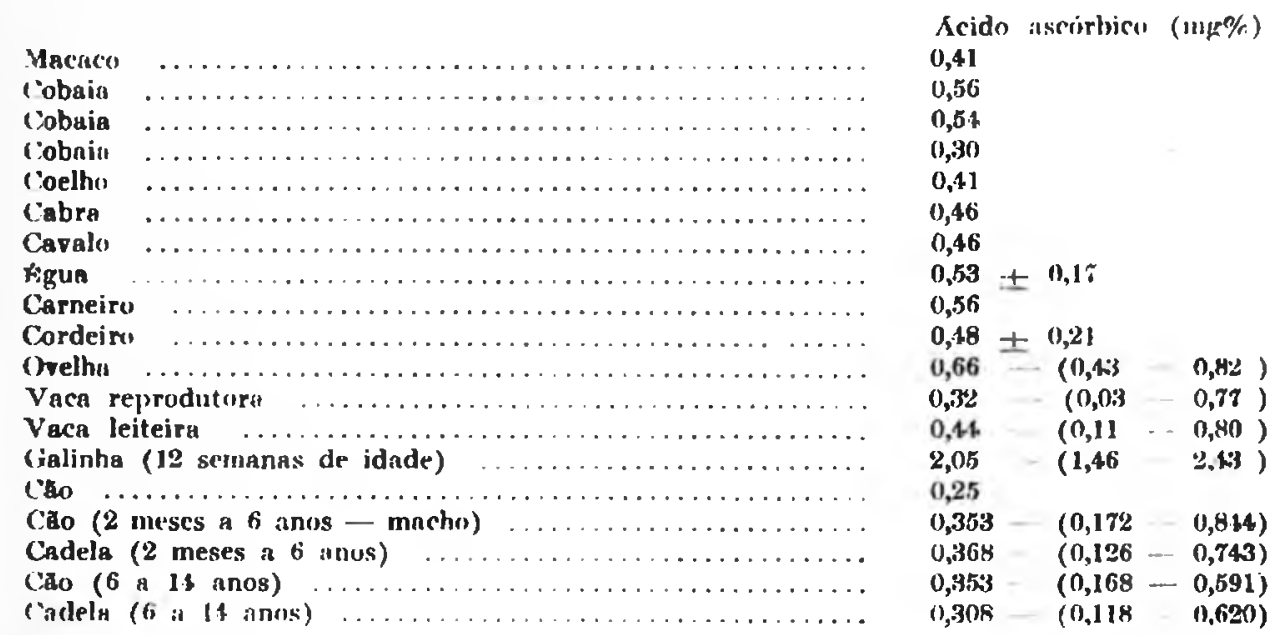




\section{R L S I M O}

Neste trabalho foi determinada a taxa média de ácido ascórbico em sangue total de eqiiinos, puro sangue de corrida. alojados o en regimem de vida hi. pica, no Jockey Cluh de. São Paulo, Brasil.

0) animais utilizados na pesquisa eram de ambos os sexos. 69 machos e 18 fêmeas e de idades variáveis entre 2 e 8 anos.

Os valôres correspondentes a animais de ambos os sexos acusaram a midia do $0,633 \pm 0,013 \mathrm{mg} \%$ de ácido ascórhico em sangue total.

Antes de reunir os dados referentes a ambos os sexos, procurou-se determinar os valôres correspondentes a machos e fêmeas em sep̧arado. Os valôrę 'ncontrados foram respectivamente $\mathrm{M}=0,638 \pm 0,017 \mathrm{mg} \%$ \& $\mathrm{Ml}=0.625 \pm$ $0.019 \mathrm{mg} \%$ de ácido ascórbico em sangue total.

A diferença entre as médias, equivalente a $0,013 \mathrm{mg} \%$, revelou-se median. le a aplicação do test $t$, deștituida de significância estalística, o que possibili. tou-mos a junção dos valôrti éstabelecidos para ambos os sexos.

Os valôres por nós obtidos para animais clinicamente sãos, em sanguc to. tal. são haixos comparados com os valôres médios para o sangue humano obtidos por 'liylor, Chase " Fallkyer e reunidos por Hawk e Bergem, com a média de $1,61 \mathrm{mg}$ por $100 \mathrm{ml}$ com uma variação de 0,8 a $2,4 \mathrm{mg}$.

Best e TAylor atribuem ao sangue humano a média normal ao redor de (),500 $\mathrm{mg}$ de ácido ascórbico em $100 \mathrm{ml}$ de sangue total; esta média daria ao valôr médio por nós encontrado uma diferença de $0,138 \mathrm{mg} \%$ para mais no sangue de eqüinos.

Dos valôres reunidos por Tonhunter e McMillix, em algumas espécies: verifica-se que somente as galinhas (Gallus gallus) apresentam teor mais elevado que os por nós apresentados. para eqiiinos.

\section{$S \quad \mathrm{U} M \mathrm{M} \Lambda \mathrm{R}$}

Ascorbic acid average levels in whole hlood of thoroughbred race horses, have been carricd out.

sixty ninc males and 18 females (which were working on races) between $2-8$ years old, were studied.

The ascorbic acid averages values for both sexes were $M=0.633 \pm 0.013$ $\mathrm{mg} \%$, being $\mathrm{M}=0.638 \pm 0.017$ for males and $\mathrm{M}=-0.625 \pm 0.019 \mathrm{mg} \%$ for females.

The difference between the averages $(0.013 \mathrm{mg} \%)$ for males and female: was vithout significance. 


\section{BIBIIOGRAFIA}

Best, H. C. - 'Axuon, N. 13. - 1950 - The physiological basis of medical practice: 748. 5th ed. Baltimore, 'The Willinms \& Wilkins Company

Cenndon, J. H. - 1.tind, C. C. - Dil, I) 13. - 19h0 - Noro kinghind J. Ifed., azs: 353

Davis, K. D. - CuLs, C. L. - 1913 - The relation of ascorbic acid to brecling performance in horses. Jour. Animal Soi., 2(1):5:3-8

HATK, P. 13. - BercenM, O. - 1937 - Practical physiolngical chenistry: 458. 11th ed.

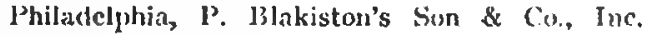

Rox, J. H. - Keturtl, C. A. - 1943 - The determination of nscorbic acid in whole blood and urine through the 2,4-dinitro-phenylhydrazinc derivative of dehydroascorbic acid. Jour. Biol. Chem., 147:399-40\%

Tromsos, W. M. - $1910 \ldots-\lambda n$ interprctation of the clinical and laboratory findings in azoturia. Comell Vet., $30: 341-9$

Todhunter, H. N. - MeHrirlax, T. J. - 1946 - The acid ascorbic content of whole blood plasma normal rats with cvidence of a sex difference. Jour. Nutrition, $31(5): 573-50$

$W_{A Y}, C .-1941$ - The importance of vitamin-mineral supplements in equine nutrition J. A. V. M. A., $99(773): 121-9$ 